\title{
MAPPING A SYSTEMS APPROACH TO EXISTING HEALTHCARE DESIGN, DELIVERY AND IMPROVEMENT METHODS
}

\author{
Günay, Aslı (1,2); \\ Ward, James (2); \\ Clarkson, P John (2) \\ 1: Koç University; \\ 2: University of Cambridge
}

\begin{abstract}
Design, delivery and improvement processes in healthcare is of great challenge due to increasing pressures on healthcare systems alongside inherent complexities, resulting in limited benefits or unforeseen consequences. A recent Systems Approach framework has aimed at responding to the calls about implementing a more holistic approach. Though being an approach in its own right that can iteratively and systematically structure, guide, and/or facilitate design and improvement projects in healthcare, it can also augment existing approaches that have already place in healthcare designers' and improvement practitioners' agendas. Thus, the objective of this paper is to compare and contrast the questions, activities, and tools of the Systems Approach with processes of other major improvement approaches in healthcare to demonstrate their coverages, overlaps, and extended and new opportunities to pursue in the light of the Systems Approach.
\end{abstract}

Keywords: Systems Engineering (SE), Lean design, Six Sigma, Multi- / Cross- / Trans-disciplinary processes, Design for Healthcare

\author{
Contact: \\ Günay, Asl1 \\ Koç University \\ Media and Visual Arts \\ Turkey \\ asgunay@ku.edu.tr
}

Cite this article: Günay, A., Ward, J., Clarkson, P. J. (2021) 'Mapping a Systems Approach to Existing Healthcare Design, Delivery and Improvement Methods', in Proceedings of the International Conference on Engineering Design (ICED21), Gothenburg, Sweden, 16-20 August 2021. DOI:10.1017/pds.2021.461 


\section{INTRODUCTION}

Designing high quality products, services and systems in all contexts and industries is of great importance, considering diverse aspects such as user experience, employee satisfaction, organisational success, environmental concerns (Langley et al., 2009); yet, it is more related even to life and death in health and care settings. Moreover, healthcare is a complex system-actually system of systems - in which the system's behaviour and performance alter over time and cannot be completely grasped by simply focusing on the individual components. Many other sectors and industries do not have such criticality, complexity, range, and breadth, regarding multiple moving parts, intricate funding models, stakeholders with diverse complicated needs, and varying interventions for a single person's needs (Braithwaite, 2018). Besides, challenges facing the health care systems are at escalation due to pressures related to increasing ageing population and accompanying multiple morbidities, depleting resources, need for seamless integration of new technologies, and quest for higher efficiencies (Clarkson et al., 2018; Government Office for Science, 2019; Powell et al., 2009; PwC, 2017; Williams, 2017). Considering the multifaceted pressures alongside inherent complexity of healthcare systems, attempts to improve healthcare systems usually result in limited benefits or unforeseen consequences. Hence, there have been calls especially in the last two decades to implement a more holistic systems approach in healthcare context, despite the lack of a clear definition of what that means in healthcare practice.

The Engineering Better Care report (Clarkson et al., 2017) has presented a new framework within the healthcare scope, grounded in a Systems Approach that is routinely used by engineers to address challenging problems in projects with diverse complexity - ranging from automobile design (e.g. Monat and Gannon, 2018) to Olympics delivery projects (e.g. Armitt, 2011) to space flight programme design (e.g. NASA, 2020). Hereby, people, systems, design, and risk emerge as the major elements of the holistic health and care design, delivery and improvement process. This holistic process is also composed of a set of questions that should be posed and answered until the current health system design improves as a measurably better one. Figure 1 (left) demonstrates the question set that delivers "what" systems approach means in healthcare.
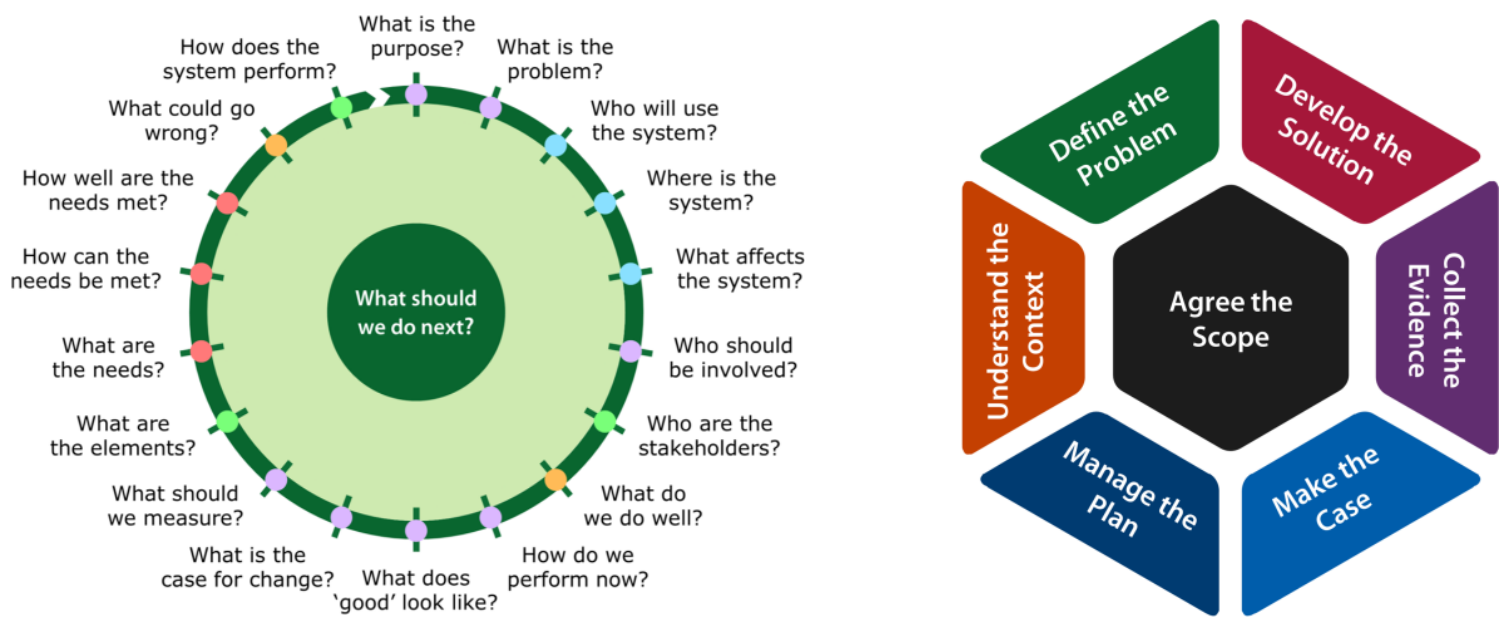

Figure 1. A systems approach to health and care design, delivery, and improvement (Clarkson et al., 2018) and the hexagon model of the Systems Approach (Clarkson, 2020)

In addition, the report has made an initial and high-level comparison and discussion of activities and tools that can be used by various stakeholders in a design and improvement team (e.g. project manager, safety manager, designer) who strive for designing and transforming health and care. Yet, a need also for prescriptive frameworks to design and redesign value effective healthcare delivery, in addition to descriptive ones, is highlighted (Ciccone et al., 2019; Patou and Maier, 2017). The building questions, activities, and tools in the explanatory report which were co-produced with engineers, clinicians, and healthcare leaders have been advanced, detailed, and concretised in a recent toolkit (Clarkson, 2020), with combined guidance and resources to operationalise the proposed systems approach in healthcare projects of any nature, scale, and levels of complexity. The healthcare design, delivery, and improvement process is structured around a hexagon model (Figure 1, right). Understand the context expresses the conditions and setting that surround a healthcare system, and 
factors that can effect its design and improvement. Define the problem explains details of a particular challenge in a system, as well as all (re)design requirements necessary to improve the system. Develop the solution describes ways of solving a certain problem in a system and (re)design components to improve the system. Collect the evidence relates to measures utilised to evidence the validity of solution(s) to the problem(s). Make the case explains facts and arguments to improve a system and deliver solutions to the problem(s). Manage the plan demonstrates a detailed proposal for delivering solution(s) to the problem(s) in the system. Agree the scope describes the context and extent of the envisaged (re)design, and the boundary of the system of interest.

It is important to note hereby that "design" in healthcare systems is mostly dealt with as "improvement" because the aforementioned critically of health issues entails a continuous and iterative process including assessment of risks, definition of performance targets, and provision of concrete evidence of the effectiveness of the design or redesign solutions in order to assess what benefits and/or harms the proposed design or redesign solution makes in relation to efficiency, safety, and stakeholder experiences (Ciccone et al., 2020; Pannunzio et al., 2019). The Systems Approach is an approach in its own right that can iteratively and systematically structure, guide, and/or facilitate such design and improvement projects in healthcare - even if this can be a redesign process of existing healthcare systems to improve their quality or designing high quality healthcare solutions from scratch. However, there are also many other approaches, theories, and tools that already have a place in healthcare designers' and improvement practitioners' agendas. Just a few examples among an extensive list of design and improvement practices in healthcare include the Model for Improvement, Lean, Six Sigma, Human Factors in Healthcare, Experience-Based Co-Design (EBCD), and Root Cause Analysis. Hence, considering the calls about and importance of pursuing a holistic systems approach in healthcare design, delivery, and improvement; but meanwhile paying regard to the importance of ongoing practices other than a systems approach, and possible difficulties in abandoning those habits due to several reasons (e.g. feeling experienced and comfortable with a certain approach, organisation culture, time to learn a new approach, etc.), we thought that identifying the components of the System Approach which are not addressed and/or used by existing approaches would also augment the existing approaches. In other words, beyond being an approach in its own right, the Systems Approach can provide a different perspective and add extra value to the existing approaches as well, by raising diversified questions in design/redesign/improvement processes and offering additional activities and tools to the former repertoires of the designers and improvers in healthcare. Thus, the aim of this paper is to compare and contrast the questions, activities, and tools of the Systems Approach with the processes of three frequently utilised improvement approaches in healthcare - IHI Model for Improvement, Lean Thinking, Six Sigma - to demonstrate their coverages, overlaps, and gaps. This literature-based mapping makes a two-fold contribution to the design field. First, it prods designers and improvers who are working or want to work in the healthcare domain to think broader than traditional design and improvement approaches and discrete methods, and challenge them to consider a more holistic approach. Secondly, by pinpointing the specific gaps, it provides a practical information source for practitioners in healthcare design, delivery, and improvement to integrate these extended and new opportunities in their ongoing processes. The following sub-section provides examples of Systems Approach in healthcare; and the next sub-section introduces other three frequently used approaches in healthcare while more details about their individual phases will be presented in relation to findings in Section 3.

\subsection{Examples of a systems approach in healthcare}

Potentials of utilising a systems approach about a wide spectrum of healthcare issues can be seen in literature; case studies ranging from medical technology design to delivering national vaccination programmes, realising clinical improvements, designing healthcare meetings, and so on. To exemplify, Ravitz et al. (2013) address the importance of a systems approach in medical device and instrumentation design, particularly in medication infusion pumps, considering the high costs, safety, and complexity of technology and care projects. Bashford et al. (2018) demonstrate how systems approach to the improvement of trauma care - which requires an understanding of the relevant people, equipment, processes, institutions, and power structures - could inform both clinical improvement and

global academic collaboration. Ciccone et al. (2019) discuss the incorporation of a systems design perspective into dementia care during which interventions are usually centred on utilising smart health 
technologies to improve healthcare systems through behaviour data collection, processing and application, targeting cognitive decline.

The evidence base for a systems approach to healthcare design, delivery, or improvement has been systematically reviewed by Komashie et al. (2021); and they observe statistically significant positive patient and service outcomes in studies that used a systems-related approach in any healthcare setting (e.g. an entire national health system, university hospitals, suburban areas, specific care units, diverse health domains) with any patients (e.g. pregnant women, parents, stroke patients, cardiac patients, adult smokers) or healthcare professionals (e.g. physicians, nurses, theatre staff, medical students, caregivers, therapists).

\subsection{Other major improvement approaches in healthcare}

\subsubsection{IHI model for improvement}

Developed by Associates in Process Improvement (API), the Model for Improvement is adopted by the Institute for Healthcare Improvement (IHI). Its use is widespread in healthcare systems in the UK and US. The model is composed of three questions that drive all improvement and the plan-do-studyact (PDSA) test cycle to test changes in real work settings in order to determine if the change is an improvement (Langley et al., 2009). The three questions are: What are we trying to accomplish? How will we know that a change is an improvement? What change can we make that will result in improvement? These are respectively related to setting aims, establishing measures, and selecting changes (IHI, 2020). The Shewhart Cycle, Deming Cycle, and plan-do-check-act (PDCA) cycle terminologies are used for the variations of the PDSA cycle. The use of the model over time for achieving improvement with the application of many small consecutive PDSA cycles is sometimes referred to as rapid-cycle improvement (Horton, 2004). It promotes a trial-and-learning approach to improvement efforts, with encouragement to test an idea rather than carry out extensive analysis (Langley et al., 2009). Its widespread use is due to this ability to bring about rapid testing of ideas, while being simple. However, there are criticisms about its effectiveness, asserting that it is usually poorly applied and pursued through time-limited, small-scale projects, led by professionals lacking the expertise, power or resources to instigate the changes required (Dixon-Woods and Martin, 2016).

\subsubsection{Lean thinking}

Lean thinking is an approach to improvement developed at Toyota in the 1950s, which was referred to as the Toyota Production System (Bevan et al., 2013; Womack and Jones, 2003). It initially came to prominence in healthcare systems through The Productive Series: Releasing Time to Care (NHS Institute for Improvement, 2012), a programme developed by the NHS Institute for Innovation and Improvement and has been the subject of many other reports on methods for improvement. Also, the Virginia Mason Medical Center in Seattle, Washington is a well-known user of lean thinking principles since the onset of the millennium. Five UK NHS Trusts have been recently piloting Virginia Mason's approach (Clarkson et al., 2020).

Lean thinking is a strategic approach that focuses on dramatically improving flow in the value stream and eliminating waste. In healthcare, it highly focuses on improving patient flow, reducing opportunities for error, developing standards, and engaging teams in improvement. So, it is fundamentally a team process involving multiple people across an organisation. It is founded on five principles designed to: specify the value desired by the user/patient; map the value stream and identify the steps that do not create value; create a smooth flow through the value-added steps; establish pull between the steps; and seek perfection so that the number of steps and the time and information needed to serve the user/patient are minimised.

Although it has strengths, such as looking at processes and value in a patient-centred way, having a bottom-up approach to change, streamlining healthcare delivery by reducing areas of waste one at a time and making the healthcare journey more pleasant especially both for the patients and healthcare professionals (Jones and Mitchell, 2006; Powell et al., 2009); it has been also subject to criticism, such as: lacking consideration for human factors and strategic perspective, relative inability to cope with variability, and its association with laying off people (Jones and Mitchell, 2006). Thus, it is increasingly used in conjunction with Six Sigma, as Lean Six Sigma in Healthcare (Bevan et al., 2013; Powell et al., 2009). 


\subsubsection{Six sigma}

Six sigma was developed at Motorola in the 1980s to eliminate defects and variations in processes. Its aim was to reach a performance level equivalent to a defect rate of 3.4 defects per million opportunities. It has been adopted by a number of US companies, whereas its use in the UK health system is relatively more limited (e.g. NHS Improvement, 2011). Six sigma has a meticulous focus on comprehending a wide range of customer needs, prioritising these, and designing processes and systems to cater those needs. To do as such, it uses a disciplined and systematic approach to look at the improvement journey from a number of related perspectives: define; measure; analyse; improve; and control (DMAIC). This is usually a facilitated process where experts use qualitative and quantitative techniques to drive process improvement. Although the tools may not be unique, the way they are applied and integrated as part of a system is. Extensive training is a must for Six Sigma professionals, to be able to select and use tools to evaluate a process from diverse perspectives and determine which activities are to be improved. Organisations involved in a Six Sigma utilise specially trained individuals with a belt system (Montgomery and Woodall, 2008).

Six Sigma tools are utilised to: define a problem, improvement opportunity, or requirements; measure process performance; analyse processes to determine root causes of variation, defects, or poor performance; improve process performance by addressing root causes; and control the improved process and future performance.

Though it is appreciated due to its rigorous methods, this is also the very reason it receives certain criticisms as this may not be always an appropriate goal. Criticism involves it being merely about quality, which might disregard the real value and not be user/patient-centric; overlooking system interaction and resulting in uncoordinated projects; being too detailed and complicated for many tasks; and necessity to rigorously train individuals (Bevan et al., 2013).

\section{MAPPING}

This work is based on the literature review and mapping process of the above mentioned three major QI approaches with the Systems Approach. These three approaches have been selected to compare and contrast with the Systems Approach due to several reasons. Firstly, healthcare systems (e.g. NHS) have apparent focus on the Model for Improvement. Secondly, there are increasing online and offline education and training sources and programs about them. Thirdly, they are also very fundamental approaches in other disciplines. Moreover, diversification in their contents and processes might be making them preferable during diverse needs in healthcare improvement projects. For the review, we considered improvement guidebooks and materials that provide information about "how" to carry out QI projects in healthcare. Individual papers that explain how they carried out improvement projects in specific projects in healthcare were beyond the scope and aim of this work. Though they were valuable to demonstrate real life examples and related learning, they might have very specific issues that cannot be generalised in such a complex system of systems. In other words, they were few papers which contained analytical reviews of the QI processes in healthcare and most of them were descriptive case studies on a single site (Boaden et al., 2008). Thus, we mainly traced resources which provide holistic guidance about how to realise improvement with the mentioned QI approaches in healthcare. These include Google and Google Scholar search, and websites of both QI and/or healthcare organisations and Foundations (e.g. The Q Foundation, NHS Institute for Innovation and Improvement, Healthcare Quality Improvement Partnership, Eastern Academic Health Science Network, Health Quality Ontario, etc.). We also targeted resources in the format of a "toolkit", "toolbox", "guide", "guidebook", and "manual" for QI in healthcare. Furthermore, thinking about the most recent strategies in QI projects in healthcare which might not yet have been delivered in the form of an extensive information resource, we perused other online and offline guidance materials and information sources that we were able to reach, such as presentation slides of training programmes on the QI approaches.

For the analysis, we firstly read through all the resources and materials, and then analysed their contents in detail to ascertain how they could relate to the Systems Approach. To structure the analysis, we used the key questions that define an iterative Systems Approach in healthcare, and activities and tools involved. So, we devised a Microsoft Excel sheet, which is a tool frequently used in social sciences and design disciplines to content analyse qualitative data (e.g. Günay and Erbuğ, 2018; Ose, 2016), and used its matrix structure to reductively organise the analysis around 24 question keywords, 42 activities, and 45 tools of the Systems Approach (the list of all can be found in Figure 2) 
in relation to three frequently used approaches in healthcare design, delivery, and improvement. In addition to extracting those ingredients of the Systems Approach from other QI approaches, the level of support provided with existing QI approaches has been examined. The findings were categorised as: issues covered in detail in the resources, mentioned in the resources without detailed guidance, and not mentioned at all. After the comparison we came up with overlaps and, importantly, gaps where the systems approach can add further value.

\section{Questions}

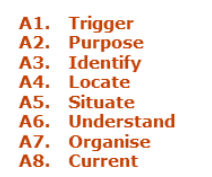

\section{Activities}

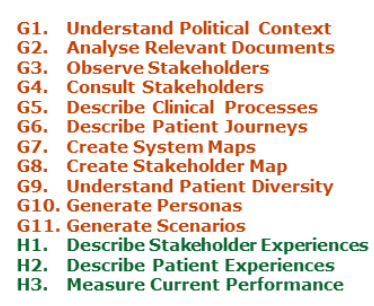

\section{Tools}

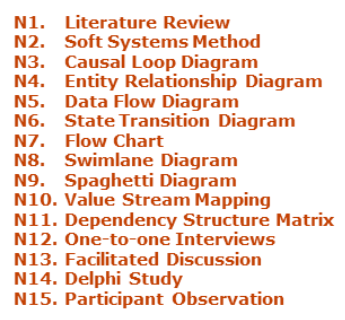

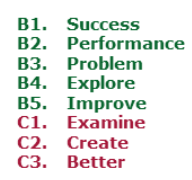

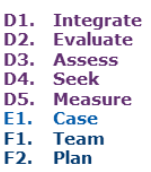

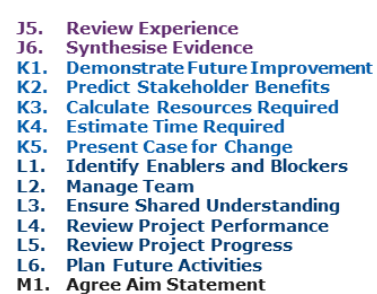

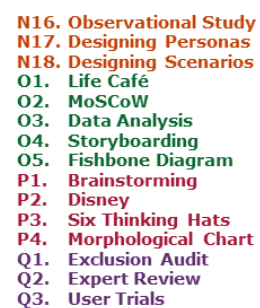

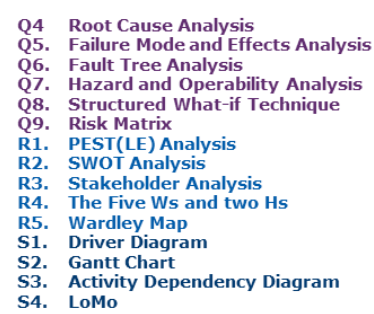

Figure 2. Questions, activities, and tools in the systems approach to healthcare (Colours match the hexagon model in Figure 1, right)

\section{RESULTS}

The results are presented around three approaches and their main stages. Each sub-section contains a summary - a mapping diagram providing a comparison of each approach with the Systems Approach. The detailed explanations of all 101 items, questions, activities, and tools are presented by Clarkson (2020). We will directly present the overlaps and gaps; and make an overall discussion in the next section. To facilitate the reading of the diagrams, Figure 1 (right) and 2 function as legends for colours and numbers in the diagrams. Three concentric rings contain respectively (from the inside out): the questions, activities, and tools. The biggest diagrams show the mapping of current conditions, i.e. support that resources provide and its level. Numbered circles' shades depict the level of support: item covered in detail in the resources (bolder shade), mentioned in the resources without detailed guidance (lighter shade), and not mentioned at all (no shading). The small diagrams that are vertically aligned on the right sides show where the Systems Approach can complement each stage of the QI approaches.

\subsection{IHI model for improvement}

What are we trying to accomplish (Aim)? The focus of the first question in reviewed resources is the agreement of a SMART aim statement that is meaningful to the users and stakeholders, and aligned with organisational goals. General project issues are also addressed at this stage, such as creation of the team, initial exploration of the problem area and needs, definition of a clear scope for the improvement, and engagement of key stakeholders.

How will we know that a change is an improvement (Measures)? The second question in reviewed resources relates to the identification and definition of appropriate measures to test ideas for improvement. These may include outcome, process and balancing measures, proposed by the stakeholders, to ensure the agreed improvement aim is met. 
What change can we make that will result in improvement (Changes)? This question addresses the development of change ideas, linked to needs and the agreed aim and measures. The use of creative thinking and exploration, along with the visualisation of current processes and evidence, are proposed as a means to stimulate ideation, concept development and selection.

PDSA Cycle: PDSA cycles initially focus on testing the proposed changes at a limited scale to reduce risk, and then on learning from subsequent cycles to inform the scale-up of the changes. Thus, PDSA appears to be also in relation to second question on measures, measurement and data, and to the third regarding the current system and processes.

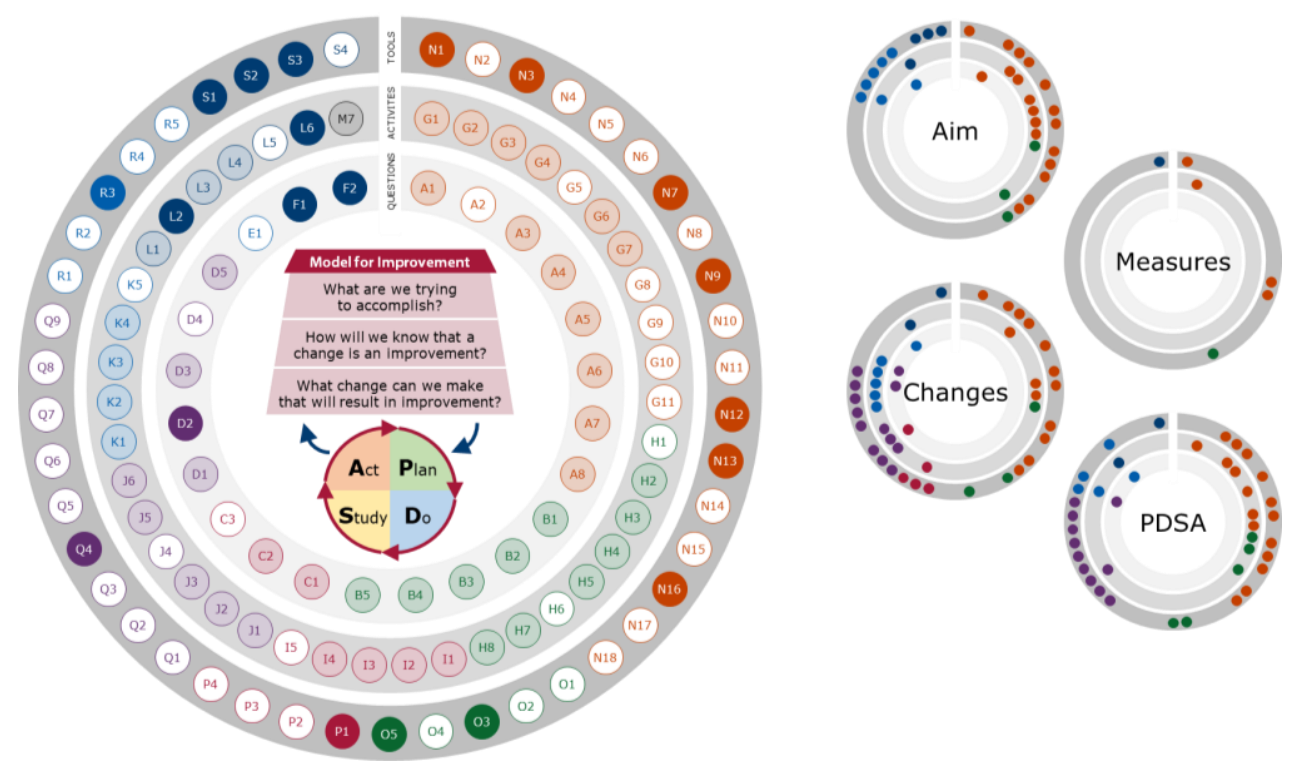

Figure 3. Outcome mapping diagram of IHI Model for Improvement

\subsection{Lean thinking}

Identify value: The first principle is usually related to general project issues: the creation of the team, definition of the project aim, scope and plans, and creation of a sense of urgency; exploration of the problem and its context; and investigation of the high-level system processes, their elements, interfaces and boundaries. There is particular focus on the definition of value from the users' perspective, through the identification of patients and other stakeholders, capture of their views, and understanding of their problems, needs and expectations.

Map the value stream: The second principle focuses on the elaboration of the high-level system processes previously captured. Process maps are used to visualise the current end-to-end processes in some detail, distinguishing value-add and non-value-add steps. For a step to add value, a user or stakeholder must care about it, it must change them or knowledge about them, and it must be executed right at the first time. If those are not met, that step is a waste. The timing of the process steps is also reviewed regarding identifiable priorities, potential bottlenecks and constraints.

Create flow: The third principle focuses on the elimination of waste, avoidance of uneven flow, batching and queuing, and establishment of a smooth flow of users or other entities in the value stream. Lean fundamentals and standard solutions to common problems are considered to develop ideas for the elimination of waste and improvement of flow. A new value stream is proposed and gaps between this and the current state identified and reviewed.

Establish pull: The fourth principle focuses on letting the users or stakeholders pull value rather than pushing it, without delay or reliance on excessive resources. Creation of a pull mechanism aligns with the need for smooth flow, which requires good evaluation of resource usage against demand, and communication and visibility of the progress being made.

Seek perfection: This principle emphasises the maintenance of gains and continuous effort for achieving perfection. The performance of the revised system and perceptions of users and stakeholders about the new practices are monitored. This involves training, measurement, documentation and visualisation to remove barriers, and sustain and spread improvements. 

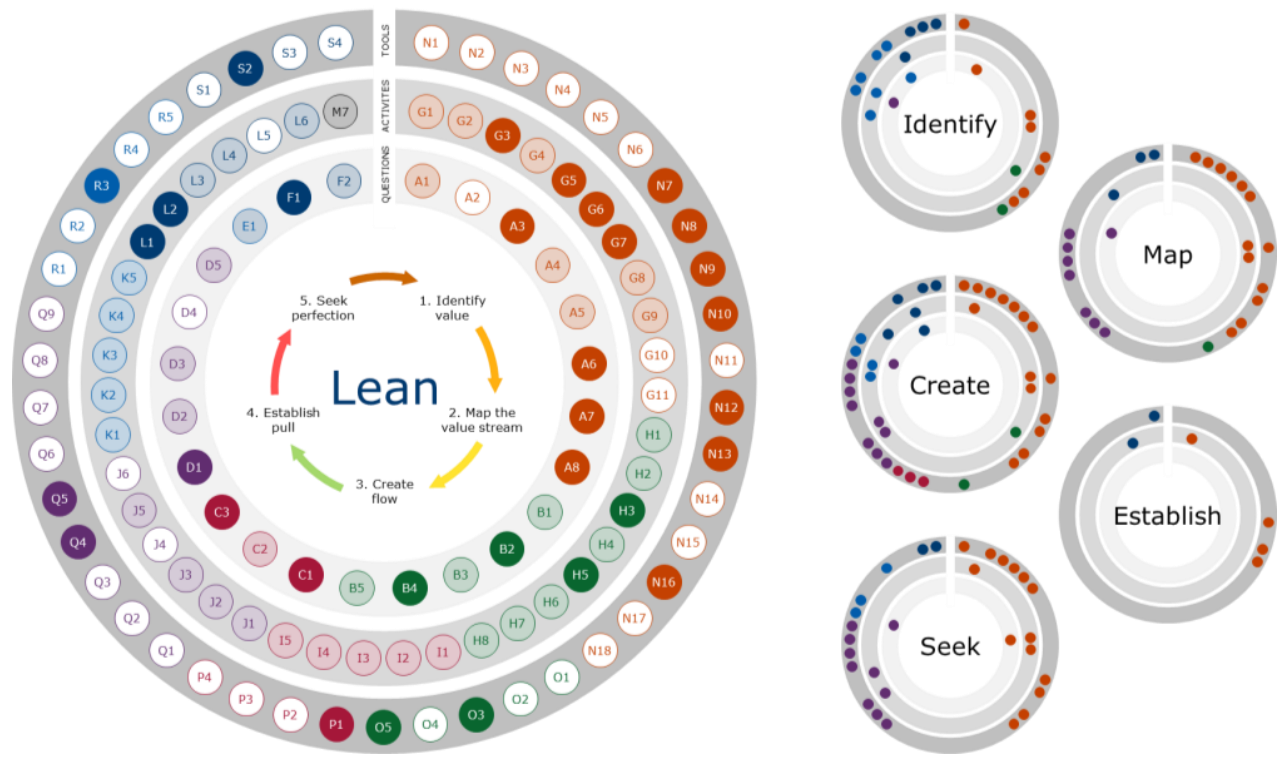

Figure 4. Mapping with Lean Thinking

\subsection{Six sigma}

Define: Clarification of the project opportunity, creation of the team, definition of the problem aim and scope, identification of the users and stakeholders, description of measures of success, and agreement of the major milestones are involved. Importance is given to the voice of users/stakeholders, and its translation to critical-to-quality trees that illustrate how needs map to actionable requirements, and facilitates understanding about the current processes and improvement opportunities. Potential benefits to the users and stakeholders are also considered in the light of the resources and time required to deliver such improvement.

Measure: The detailed requirements and techniques for measurement of the current system performance are usually established to capture a true picture of the process. This includes the identification of measures of variance and different data types, definition of key process input and output variables, development of a data collection plan, and an initial data analysis.

Analyse: The third stage focuses on the detailed analysis of system performance data, examination of different sources of variability, determination of cause and effect relationships and root causes of problems, and identification of specific opportunities for improvement.

Improve: This stage involves creative thinking and the generation of improvement concepts that address the root causes of the variations and problems observed. These concepts are subsequently evaluated, prioritised, piloted and implemented. A future-state system process map is used to visualise the impact of the proposed changes, identify the gaps between the current and future states, and enable risk assessment of the proposed system. Cost/benefit analysis and planning for full-scale implementation are also undertaken at this stage.

Control: The final stage focuses on institutionalising and controlling the improved processes. This includes process monitoring, preparation of training documents, control and reaction plans, and documentation of before and after results and updated process maps.

\section{DISCUSSIONS AND CONCLUSION}

Beyond its natural importance due to its direct relevance to health, and life and death, improving quality in healthcare is getting more and more crucial concerning the pressures on healthcare systems and inherent complexity of this system of systems. Practitioners in healthcare design, delivery, and improvement already have a valuable repertoire of methods, activities, and tools. Considering the importance of following a holistic systems approach, we examined how a Systems Approach can provide a different perspective and add extra value to the existing approaches. Findings show that three of the frequently used improvement approaches in healthcare address many key questions, but frequently they do not provide detailed guidance (see the lighter shades in the inner rings of the biggest diagrams in Figure 3-5). Looking at different categories of these key questions, Lean resources provide relatively more concrete guidance to practitioners; particularly on: "understand the context", 
"define the problem", and "develop the solution" strands in the hexagon model. Model for Improvement resources provide more detailed guidance about the key questions related to the "manage the plan" strand. For Six Sigma, guidance on how to "collect the evidence" is provided to a certain extent. Resources do not lead to description or guidance on defining the purpose of the system and seeking to understand what is done well in the current system, which can lead to a systematic assessment of the presence or potential impact of opportunities, beyond problems and risks. Thus, a Systems Approach can complement existing approaches firstly on these two questions, and further enhance them by providing details and guidance about existing explanations.
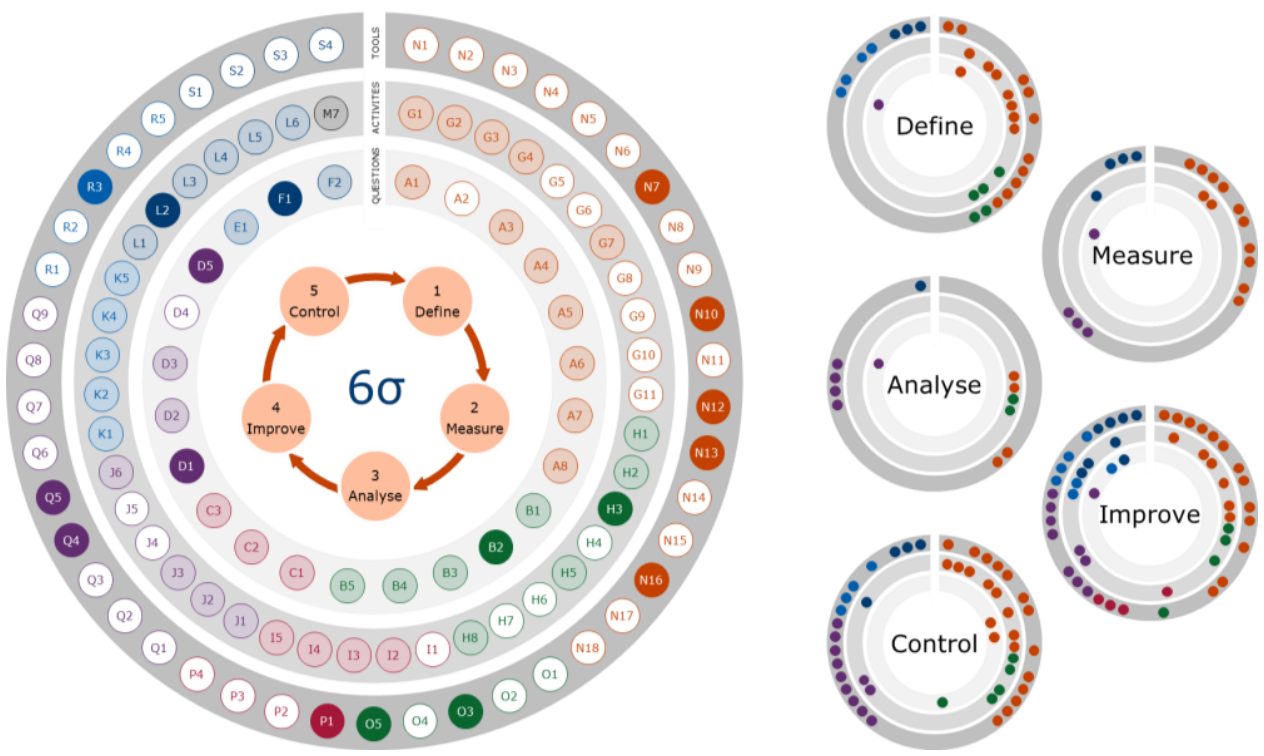

Figure 5. Mapping with Six Sigma

Almost all resources do not provide any guidance on how to carry out activities (the middle rings), whereas guidance on more specific tools can be found (the outer rings). However, the challenge is also to select and match the tools offering detailed guidance with the appropriate activities in which they can be used. Also, existing resources do not draw on a wide range of tools that we compiled. The systems approach can add particular value by encouraging practitioners to consider also those diverse tools which might be more appropriate to their aims and boundaries in different projects.

When other improvement approaches provide descriptions without detailed guidance, the Systems Approach concretises these descriptions and support them with guidance. For the common coverages about which other approaches have clear guidance, the questions, activities, and tools in the Systems Approach can still serve the function of a reminder, facilitator, and accelerator by presenting a pool of thoughts, actions, and examples. All these issues are not exhaustive and strict, practitioners who aim for designing, delivering, and improving healthcare can use and explore them in a flexible way.

This work has its limitations. The paper is based on a literature review, which we believe is very beneficial to create a fruitful conversation point between improvement practitioners and system specialists. Yet, it is also important to go beyond and corroborate the positioning of the Systems Approach in practice by quality improvers. Also, more case studies are needed to evaluate how healthcare designers and improvers which are not currently using a systems approach would integrate the addressed extended possibilities in their ongoing practices. We are planning further studies with the current learning from the literature.

\section{REFERENCES}

Armitt, J. (2011), Engineering the Olympics: The 2011 Lloyd's Register Educational Trust Lecture. Royal Academy of Engineering.

Bashford, T., Myint, P.P.N., Win, S., Thu, M., Naing, M.M., Burnstein, R., Hlaing, T.T., Brealey, E., Hutchinson, P. and Clarkson, J. (2018), “A systems approach to trauma care in Myanmar: from health partnership to academic collaboration”, Future Healthcare Journal, Vol. 5 No. 3, pp. 171-175.

Bevan, H., Westwood, N., Crowe, R. and O’Connor, M. (2013), Lean Six Sigma: Some Basic Concepts, NHS Institute for Innovation and Improvement. 
Boaden, R., Harvey, G., Moxham, C. and Proudlove, N. (2008), Quality Improvement: Theory and Practice in Healthcare, NHS Institute for Innovation and Improvement, Coventry.

Braithwaite, J. (2018), "Changing how we think about healthcare improvement", BMJ (Clinical research ed.), Vol. 361 No. k2014. https://doi.org/10.1136/bmj.k2014

Ciccone, N., Patou, F. and Maier, A.M. (2019), "Designing for Better Healthcare: A Systemic Approach Utilising Behavioural Theory, Technology and an Understanding of Healthcare Delivery Systems", ICED19, Delft, The Netherlands, 5-8 August 2019, pp. 937-946. http://doi.org/10.1017/dsi.2019.98

Ciccone, N., Patou, F., Komashie, A., Lame, G., Clarkson, P.J. and Maier, A.M. (2020), "Healthcare Systems Design: A Sandbox of Current Research Themes Presented in an International Meeting", International Design Conference - DESIGN 2020, Online, 26-29 October 2020, Cambridge University Press, 1, pp. 1873-1882. https://dx.doi.org/10.1017/dsd.2020.24.

Clarkson, J. (2020), Improving Improvement Toolkit. [online] Cambridge Engineering Design Centre. Available at: www.iitoolkit.com

Clarkson, J., Bogle, D., Dean, J., Tooley, M., Trewby, J., Vaughan, L, Adams, E., Dudgeon, P., Platt, N. and Shelton, P. (2017), Engineering Better Care, a systems approach to health and care design and continuous improvement, Royal Academy of Engineering, London, UK.

Clarkson, J., Dean, J., Ward, J., Komashie, A. and Bashford, T. (2018), “A systems approach to healthcare: from thinking to practice", Future Healthcare Journal, Vol. 5 No. 3, pp. 151-155.

Dixon-Woods, M. and Martin, G.P. (2016), “Does quality improvement improve quality?”, Future Hospital Journal, Vol. 3 No. 3, pp. 191-194.

Government Office for Science (2019), Future of an Ageing Population. Available at: www.gov.uk/government/publications/future-of-an-ageing-population

Günay, A. and Erbuğ, Ç. (2018), "Mobile Health Integration in Pregnancy”, In: Sezgin, A., Yildirim, S., ÖzkanYilirim, S. and Sumuer, E. (Eds), Current and Emerging mHealth Technologies, Springer, pp. 57-83.

Horton, S. (2004), "Increasing capacity while improving the bottom line", Frontiers of Health Services Management, Vol. 20 No.4, pp. 17-23.

Institute for Healthcare Improvement (2020), Science of Improvement: How to Improve. [online] Available at: http://www.ihi.org/resources/Pages/HowtoImprove/ScienceofImprovementHowtoImprove.aspx

Jones, D. and Mitchell, A. (2006), Lean thinking for the NHS, The NHS Confederation, London.

Komashie, A., Ward, J., Bashford, T., Dickerson, T., Kaya, G.K., Liu, Y., Kuhn, I., Günay, A., Boddy, N., O'Kelly, E., Masters, J., Dean, J., Meads, C. and Clarkson, P.J. (2021), "Systems approach to health service design, delivery and improvement: a systematic review and meta-analysis”, BMJ Open, Vol. 11 No. 1. http://dx.doi.org/10.1136/bmjopen-2020-037667

Langley G.J., Moen R.D., Nolan K.M., Nolan T.W., Norman C.L. and Provost L.P. (2009), The Improvement Guide: A Practical Approach to Enhancing Organizational Performance (2nd edition), Jossey-Bass Publishers, San Francisco.

Monat, J.P. and Gannon, T.F. (2018), “Applying Systems Thinking to Engineering and Design”, Systems, Vol. 6 No. 34.

Montgomery, D.C. and Woodall, W.H. (2008), "An Overview of Six Sigma, International Statistical Review", Vol. 76, No. 3, pp. 329-346. https://doi.org/10.1111/j.1751-5823.2008.00061.x

NASA (2020), Systems Engineering Handbook (Revision 2). Available at: www.nasa.gov/connect/ebooks/nasasystems-engineering-handbook

NHS Improvement (2011), Plan, Do, Study, Act (PDSA) cycles and the model for improvement. Available at: https://improvement.nhs.uk/documents/2142/plan-do-study-act.pdf

NHS Institute (2012), Putting patients first-The Productive Series, NHS Institute for Innovation and Improvement, Coventry.

Ose, S.O. (2013), "Using Excel and Word to Structure Qualitative Data, Journal of Applied Social Science”, Journal of Applied Social Science, Vol. 10 No. 2, pp. 147-162. https://doi.org/10.1177/1936724416664948

Pannunzio, V., Kleinsmann, M. and Snelders, D. (2019), "Three Approaches to Design Engineering in the Health Domain: A Systemic Perspective", ICED19, Delft, The Netherlands, 5-8 August 2019, pp. 10051014. https://doi.org/10.1017/dsi.2019.106

Powell, A.E., Rushmer, R.K. and Davies, H.T.O. (2009), A systematic narrative review of quality improvement models in health care, NHS Quality Improvement Scotland.

PwC (2017), Emerging Trends in Healthcare: Depleting Resources Adding Pressure to Healthcare. Available at: www.pwc.com/gx/en/industries/healthcare/emerging-trends-pwc-healthcare/depleting-resources.html

Ravitz, A., Sapirstein, A., Pham, J.C. and Doyle, P.A. (2103), "Systems Approach and Systems Engineering Applied to Health Care: Improving Patient Safety and Health Care Delivery", John Hopkins Apl Technical Digest, Vol. 31 No. 4, pp. 354-365.

Williams, S.J. (2017), Improving Healthcare Operations: The Application of Lean, Agile and Leagility in Care Pathway Design, Palgrave Pivot, Cham.

Womack, J.P. and Jones, D.T. (2003), Lean Thinking: Banish Waste and Create Wealth in Your Corporation (Revised and Updated). New York, Free Press. 